\section{La Révolution française}

Cahiers de l'Institut d'histoire de la Révolution française

$16 \mid 2019$

1801-1840 - Haïti, entre Indépendance et

Restauration

\title{
Refuge et sociabilité politique : les francs-maçons domingois aux États-Unis au début du XIX ${ }^{\mathrm{e}}$ siècle
}

\section{Marieke Polfliet}

\section{(2) OpenEdition \\ 1 Journals}

Édition électronique

URL : http://journals.openedition.org//rf/2788

DOI : $10.4000 /$ Irf. 2788

ISSN : 2105-2557

Éditeur

IHMC - Institut d'histoire moderne et contemporaine (UMR 8066)

Référence électronique

Marieke Polfliet, «Refuge et sociabilité politique : les francs-maçons domingois aux États-Unis au début du XIXe siècle », La Révolution française [En ligne], 16 | 2019, mis en ligne le 20 juin 2019, consulté le 22 juin 2019. URL : http://journals.openedition.org/lrf/2788; DOI : 10.4000//rf.2788

Ce document a été généré automatiquement le 22 juin 2019

(c) La Révolution française 


\title{
Refuge et sociabilité politique : les francs-maçons domingois aux États- Unis au début du XIX ${ }^{\mathrm{e}}$ siècle
}

\author{
Marieke Polfliet
}

1 L'Atlantique français subit, à l'orée du $\mathrm{XIX}^{\mathrm{e}}$ siècle, des recompositions liées aux révolutions et aux guerres en Europe et dans les Antilles. Le tournant du XIx ${ }^{e}$ siècle voit la perte définitive des principaux atouts de l'empire colonial français aux Amériques, d'une part, par l'échec de l'expédition Leclerc à Saint-Domingue, la victoire des esclaves insurgés et la proclamation de l'indépendance d'Haïti ; et, de façon concomitante, par la cession de la Louisiane aux États-Unis. L'afflux de réfugiés d'origine domingoise dans les ports américains à partir des années 1790 va de pair avec leur regroupement, plus ou moins informel, en fonction des liens familiaux, amicaux ou d'affaires, du fait de la commune nécessité de recourir à toutes les ressources dans un contexte d'incertitude et de difficultés matérielles. Les expériences antérieures de mobilité dans le contexte colonial et les réseaux français établis au sein de l'Atlantique au XVIII ${ }^{\mathrm{e}}$ siècle expliquent la rapidité avec laquelle les réfugiés reconstituent en exil des formes de sociabilité issues des brassages qui interviennent dans leurs différents lieux de refuge temporaire ${ }^{1}$. Au tournant du siècle, les migrations de refuge ont donné lieu à l'émergence des premières structures - journaux, loges maçonniques - destinées à recréer un lien entre les réfugiés.

Or Saint-Domingue a constitué, à la veille de la Révolution, un pôle d'expérimentation et de diffusion de formes de sociabilité nouvelles au sein desquelles la franc-maçonnerie a joué un rôle majeur. En effet, la circulation des pratiques maçonniques entre l'Europe et les Antilles au XVIII ${ }^{\mathrm{e}}$ siècle a contribué à l'organisation de nouveaux rites, tels les hauts grades du rite Écossais Ancien et Accepté progressivement formalisés par Étienne Morin lors de ses déplacements à Saint-Domingue et dans les Antilles dans les années 1760-1770² . Le transfert de ces grades aux États-Unis puis en France par l'amiral de Grasse-Tilly lors de la période révolutionnaire prolonge cette forme de rayonnement ${ }^{3}$. Cependant, dans le cadre du renouvellement récent des recherches sur la franc-maçonnerie antillaise ${ }^{4}$, les continuités et les ruptures dans les solidarités maçonniques dans les Antilles, en 
particulier après l'indépendance d'Haïti, sont encore à explorer. C'est pourquoi l'étude de ce phénomène du point de vue des lieux de refuge américains des francs-maçons réfugiés de Saint-Domingue permet de mettre en lumière les prolongements du rayonnement maçonnique domingois, à travers les incertitudes et les étapes de leur nouvel ancrage dans les ports américains au début du XIX ${ }^{e}$ siècle.

\section{Saint-Domingue, centre de sociabilité maçonnique atlantique dans la période pré-révolutionnaire}

3 La perle des Antilles est, au XVIII siècle, un espace d'intense développement de la sociabilité propre à l'époque des Lumières, voire un lieu de diffusion et d'innovation notamment dans le cadre de la franc-maçonnerie. À l'époque des Lumières, des relations maçonniques intenses se sont établies entre les Antilles, le continent américain et l'Europe. Un "espace maçonnique atlantique ${ }^{5}$ " émerge, dont les négociants qui traversent l'Atlantique sont des vecteurs privilégiés. Des liens unissent en particulier les loges des villes portuaires françaises et celles des colonies antillaises ${ }^{6}$.

4 Les migrations entre la France et ses colonies américaines - mais plus largement, en direction des Amériques - ont en effet connu une phase croissante dans la deuxième moitié du XVIII ${ }^{e}$ siècle. À Saint-Domingue, au sein la population blanche d'environ 28000 à 30000 personnes dans la partie française de l'île à la veille de la Révolution, la moitié est née en France ${ }^{7}$. Des liens familiaux et commerciaux, des chaînes migratoires se sont ainsi tissés tout au long $\mathrm{du} \mathrm{xvIII}^{\mathrm{e}}$ siècle à travers l'Atlantique. Certaines régions métropolitaines, telles que le Sud-Ouest et la Bretagne, sont des bassins d'émigration particulièrement marqués ${ }^{8}$. Les colons issus des ports de la façade atlantique française reconstituent ainsi des liens familiaux, professionnels, d'interconnaissances de l'autre côté de l'Atlantique. Bordeaux, Nantes, ou Le Havre sont des plaques tournantes qui orientent les colons à la fois géographiquement et professionnellement.

5 L'exemple des échanges entre les loges maçonniques des grands ports atlantiques français avec les loges des colonies françaises aux Amériques confirme l'existence de circulations et d'interconnaissances dans les milieux des marchands, des capitaines de navires, des marins ou des membres de l'administration coloniale9. Pierre-Yves Beaurepaire a par ailleurs souligné les pratiques d'autonomie qui se développent dans ces ports français visà-vis des autorités parisiennes et de la vocation centralisatrice du Grand Orient. Cette autonomie se retrouve dans les colonies, où se développent des pratiques spécifiques et distinctes de la métropole, par exemple dans le traitement des différences raciales, même si les pratiques de hiérarchisation sociale entre loges ne sont pas très différentes de la situation française ${ }^{10}$.

6 La "perle des Antilles» est un pôle majeur de diffusion de la franc-maçonnerie, comptant, en 1789, 22 loges ainsi qu'une Grande Loge Provinciale, regroupant au moins 530 maçons (carte 1$)^{11}$. 


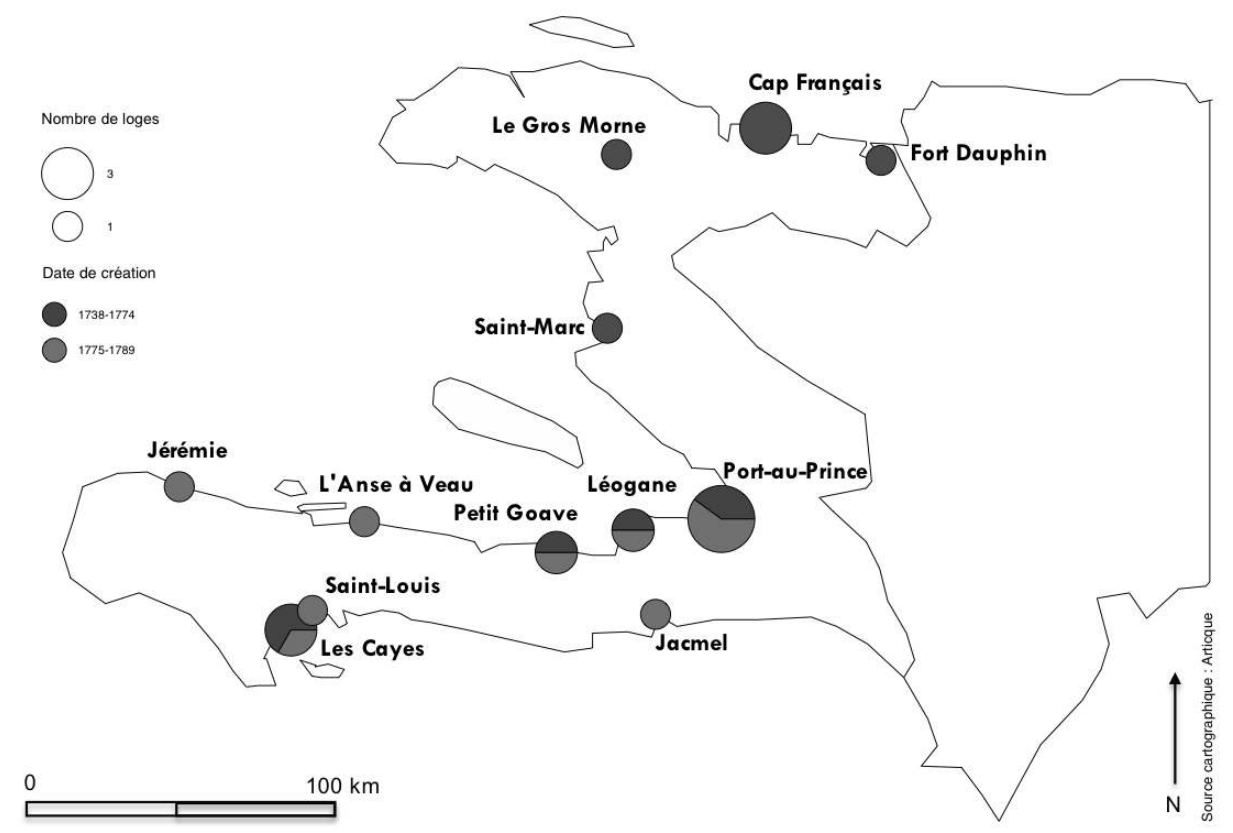

Carte : L'implantation des loges maçonniques à Saint-Domingue à la fin du XVIII siècle.

Source : GEORGES Odo, La franc-maçonnerie dans les colonies, 1738-1960, Paris, Éditions Maçonniques de France, 2001.

7 Le fait maçonnique à Saint-Domingue s'insère dans une société où les pratiques de sociabilité politique sont très avancées, comme en témoignent les clubs, cercles et journaux qui se sont formés dans la colonie ${ }^{12}$. Jacques de Cauna rappelle la complexité des affiliations maçonniques à Saint-Domingue, où il estime que la part de francs-maçons dans la société blanche est jusqu'à dix fois supérieure à celle qui existe en France ${ }^{13}$. La franc-maçonnerie se diffuse donc essentiellement dans les centres urbains, mais présente des profils sociologiques et politiques diversifiés : Élizabeth Escalle et Mariel GouyonGuillaume distinguent ainsi les loges de campagne, aristocratiques, réunissant de grands propriétaires, des loges urbaines, de composition sociale modeste, ouvertes aux artisans et employés, et des loges de notables, plus élitistes, à l'image du "Cercle des Philadelphes » au Cap, auquel appartient Moreau de Saint-Méry ${ }^{14}$. Les francs-maçons se trouvent partagés entre mouvances politiques et appartenances sociales diverses, bien que, dans le contexte colonial, l'une des caractéristiques dominantes reste l'exclusion des personnes de couleur ${ }^{15}$. La franc-maçonnerie domingoise $d u$ XVIII $^{\mathrm{e}}$ siècle a ainsi su dessiner un espace social original, adapté à la mobilité des individus, et dont la plasticité permet de traverser des contextes difficiles, notamment les épisodes révolutionnaires ${ }^{16}$.

\section{Les migrations de refuge depuis Saint-Domingue de 1791 à 1809}

Lorsque les troubles révolutionnaires touchent Saint-Domingue de 1791 à 1803 - voire 1809 pour les Caraïbes -, les populations domingoises se trouvent éparpillées au sein de l'espace atlantique et nombre d'entre elles trouvent refuge aux États-Unis - aussi bien colons, que libres de couleur et esclaves partis avec eux. Leur présence dans les ports 
américains s'accompagne du transfert des formes de sociabilité qui prospéraient à SaintDomingue avant la Révolution parmi les colons d'origine européenne.

9 Suite à la révolte des esclaves de 1791, la situation politique à Saint-Domingue se détériore - notamment avec l'incendie du Cap-Français en 1793 -, les colons blancs, mais également des libres de couleur et des esclaves, se dispersent dans des lieux de refuge proches et plus ou moins connus ${ }^{17}$. Leurs principaux points de chute se trouvent sur la côte est des États-Unis, le golfe du Mexique et les Antilles voisines, notamment Cuba qui accueille jusqu'à 18000 personnes venues de Saint-Domingue ${ }^{18}$. Certains s'orientent également vers la métropole. Cependant, ces premières vagues sont souvent temporaires. Si le contexte révolutionnaire français et le début des guerres européennes rendent plus difficile le retour en France, les retours à Saint-Domingue et les tentatives de reprendre possession des propriétés, en revanche, sont nombreux ${ }^{19}$. Après 1802-1803 et l'échec puis l'abandon définitif de l'entreprise de reconquête française, de nouveaux flux se dirigent vers le continent américain et, toujours, vers l'̂̂le proche de Cuba, qui offre des perspectives de développement de l'économie de plantation dans lequel les Domingois ont développé un savoir-faire, notamment la canne à sucre ${ }^{20}$. Cependant, après l'invasion française de l'Espagne en 1808, d'abord victorieusement repoussée, les revers de la couronne espagnole en 1809 suscitent une vague de francophobie et poussent les autorités de Cuba à expulser la population française de l'île $e^{21}$. Cette dernière vague, concentrée dans le temps (mai-juin 1809) et dans l'espace, provoque un débarquement massif à la Nouvelle-Orléans de presque 10000 personnes, plutôt que dans les autres ports du Sud, l'arrivée des libres de couleur et esclaves étant tolérée à La Nouvelle-Orléans et refusée ailleurs ${ }^{22}$.

10 Les points de chute des réfugiés ne sont pas choisis au hasard, comme le montre Darrell Meadows, mais répondent à des liens, forts ou faibles, tissés dans l'espace caraïbe et sur le continent américain par le biais de relations commerciales, familiales ou amicales ${ }^{23}$. Les estimations de la présence française aux États-Unis dans les années 1790 vont jusqu'à 45000 personnes, réfugiés de Saint-Domingue et émigrés royalistes confondus ${ }^{24}$. Malgré la diversité sociale, la forte mobilité et la dispersion de ces colons, la communauté de destin créée par le traumatisme de la révolte des esclaves et de la révolution haïtienne, associée à la création d'une catégorie administrative de "réfugié de Saint-Domingue » ouvrant droit aux secours de l'État français, puis à une indemnité, a mené à la désignation de ce groupe comme une « diaspora ${ }^{25}$ ». Des itinéraires individuels, tel celui de l'avocat Casimir Louis Moreau Lislet, natif de Dondon, à proximité du Cap, reflètent cette grande mobilité à travers l'Atlantique : s'étant formé au droit à Paris, il rentre à Saint-Domingue au tout début de la Révolution, fuit avec sa famille à Philadelphie en 1794, tente une réinstallation dans l'île à la fin des années 1790, mais doit fuir à nouveau en 1803 lors de l'échec final de l'expédition française. Il trouve refuge temporairement à Cuba et part très vite se fixer à La Nouvelle-Orléans en 1804, où il devient une figure centrale de la communauté domingoise. Si son itinéraire maçonnique à Saint-Domingue est mal connu, il devient en revanche un pilier dans la réorganisation des loges à La Nouvelle-Orléans au début du XIX ${ }^{e}$ siècle. 


\section{Les refondations de loges domingoises aux États- Unis, recours pour les réfugiés dans une période troublée (1791-1809)}

11 New York, La Nouvelle-Orléans, mais aussi Philadelphie ou Charleston, représentent des pôles de reconfiguration des liens maçonniques pour les Domingois qui trouvent refuge dans ces villes. La fonction d'entraide est accrue par la situation de crise vécue par émigrés ou réfugiés, qui ont perdu tout ou partie de leurs ressources avec les heurts révolutionnaires. La franc-maçonnerie s'affirme comme référent offrant des liens de fraternité et de reconnaissance, une activité de charité et de bienfaisance à l'égard de ses membres et de leurs proches, mais aussi du reste de la société des migrants.

La chronologie et l'évolution des loges maçonniques dans des grands pôles de réception des réfugiés comme La Nouvelle-Orléans et New York reflète la situation d'entre-deux dans laquelle il se trouvent, entre refuge temporaire puis ancrage et adaptation au contexte américain. Dans les années 1790, les fondations ou refondations de loges françaises sont notamment liées aux interactions des Domingois avec d'autres réfugiés de plus ou moins longue date, tels que les émigrés royalistes ou les huguenots. Ces loges, souvent conçues comme expédients provisoires, connaissent une volatilité importante des membres, mais se pérennisent néanmoins. La plasticité de la franc-maçonnerie vis-àvis des événements révolutionnaires, qui offre des ressources alternatives aux affiliations politiques ou sociales traditionnelles, explique notamment la rapidité de reconstitution des liens maçonniques dans les lieux de refuge temporaire. La refondation des loges en fait «des marques de repères voyants et pertinents ${ }^{26}$ " pour des individus dont les itinéraires ont été bouleversés.

13 La franc-maçonnerie a émergé depuis la deuxième moitié $\mathrm{du}$ XVIII ${ }^{\mathrm{e}}$ dans tous les ports de la côte atlantique américaine, y compris à La Nouvelle-Orléans et à New York ${ }^{27}$. Cependant, au-delà de ces phases pionnières, le développement de loges françaises pérennes dans les deux villes fait suite aux troubles révolutionnaires en France et à SaintDomingue. Leur influence dans le dynamisme maçonnique de la fin du XvIII siècle est déterminante, puisque toutes les loges françaises fondées à New York comme à La Nouvelle-Orléans dans les années 1790 le furent par des réfugiés français. Ces réunions sont généralement spontanées, à l'initiative d'un petit nombre de réfugiés, et leur régularisation par une autorité maçonnique n'a lieu qu'a posteriori, quelques mois, voire quelques années après leur fondation. Le chapitre de hauts grades La Triple Union de New York évoque ainsi le contexte de sa fondation ${ }^{28}$ :

Le $3^{\mathrm{e}}$ jour du $10^{\text {me }} \mathrm{MM} \therefore$ de l'an de la $\mathrm{V} \therefore \mathrm{L} \therefore 5797$ [ 3 décembre 1797 ], époque où

les premiers malheurs qui avaient affligé l'infortunée colonie de Saint-Domingue

avaient forcé nombre de ses habitans de se réfugier pour leur sûreté aux États-Unis, parmi eux se trouvoient plusieurs Maçons revêtus de hauts grades de l'ordre ayant le désir de se réunir entre eux en leurs qualités ils formèrent un Chapitre ${ }^{29}$.

14 Ces débuts sont tâtonnants, allant de chartes provisoires en interruptions. Lorsque l'émigration groupée de maçons originaires d'une même ville permet de reconstituer des loges, celles-ci sont relocalisées et considérées comme «ambulantes ». C'est le cas de $L a$ Réunion des Coeurs, fondée en 1786 à Jérémie et qui se reconstitue à Santiago de Cuba entre 1806 et 1809. La Réunion Désirée est également transplantée du Cap-Français à La NouvelleOrléans en $1806^{30}$. De même, à New York, la reconstitution de loges françaises est validée 
par les membres de la «Grande Loge Provinciale de Saint-Domingue séante provisoirement à New York ». Dans le cas où l'on ne peut pas reconstituer une loge préexistante, notamment du fait de l'hétérogénéité de leurs origines, des maçons issus de loges différentes en créent une nouvelle. Onze maçons français adressent ainsi une pétition à la Grande Loge de l'État de New York le 4 septembre 1793. Sept d'entre eux détiennent des certificats de loges de Saint-Domingue et trois de France, le dernier ayant été initié à New York ${ }^{31}$. La Loge La Tendre Amitié Franco-Américaine ainsi formée fonctionne six mois, jusqu'en 1794, et compte vingt-neuf membres. Une nouvelle pétition en 1795 donne lieu à la fondation de L'Unité Américaine, puis de L'Union Française en $1797^{32}$.

À la Nouvelle-Orléans, La Parfaite Union et L'Étoile Polaire sont fondées entre 1793 et 1794. Dans le contexte local d'une tutelle coloniale espagnole peu favorable, les fondateurs doivent s'adresser à des autorités maçonniques extérieures, en France ou aux États-Unis ${ }^{33}$ . L'Étoile Polaire est fondée par sept maçons, dont deux originaires de loges antillaises et les cinq autres de loges des principaux ports français. Elle obtient une charte provisoire de la loge La Parfaite Sincérité de Marseille en 1796 avant d'être régularisée par le Grand Orient de France en $1803^{34}$. Quant aux maçons fondateurs de La Parfaite Union en 1793, ils se tournent vers la Grande Loge de Caroline, ce qui laisse à penser qu'ils sont davantage proches des milieux huguenots, Charleston étant également l'un des pôles de refuge huguenot et de diffusion de la maçonnerie française en Amérique ${ }^{35}$.

On peut expliquer l'attachement de nombre des réfugiés français à la franc-maçonnerie par l'espace d'interconnaissances et de ressources qu'elle procure. La constitution des loges, comme l'a souligné Éric Saunier dans le cas normand, est d'abord et souvent l'affaire des « hasards des rencontres ${ }^{36}$ » entre individus, qui permettent l'agrégation d'un noyau de base, auquel viennent se greffer ensuite des éléments attirés par des solidarités familiales, régionales ou professionnelles. La fonction de structures de rassemblement, mais aussi de secours et de solidarité, est bien visible à travers les correspondances, ainsi que les minutes des différentes loges, qui témoignent de l'usage courant du tronc des pauvres afin de venir au secours de réfugiés, notamment veuves et orphelins, mais également par la dispense du paiement des quotités pour les frères dans le besoin ${ }^{37}$. Les loges deviennent ainsi des points de ralliement des réfugiés de Saint-Domingue, notamment les deux loges La Concorde et La Persévérance créées en 1810 à La NouvelleOrléans suite à l'afflux de réfugiés venus de Cuba. Pierre Pajaud, né à Jérémie, membre de La Réunion des Cours, reconstituée à Cuba, obtient ainsi de Julien Bayard, l'ancien Vénérable de la loge, l'autorisation de se joindre à La Persévérance, loge qu'il a choisie, ditil, « parce qu'elle réunit tous mes amis ou mes connaissances ${ }^{38}$ ». Les loges apparaissent comme le recours des réfugiés lorsque le reste de leurs repères a disparu : les membres de La Persévérance rappellent que la reprise des travaux au sein de la loge « [les] aida à sortir de l'abattement où [les] avaient plongé le malheur qu'[ils] ven[aient] d'essuyer en l'isle de $\mathrm{Cuba}^{39} »$.

17 Si les premières loges créées présentent un caractère temporaire et une forte volatilité de leurs membres, elles deviennent dans un second temps des structures d'intégration d'un nombre croissant de réfugiés des deux villes. 


\section{Les interactions entre franc-maçonnerie et politique et l'adaptation des loges françaises au cadre américain au début du XIX ${ }^{\mathrm{e}}$ siècle}

Bien que s'adressant à une frange restreinte de la population des réfugiés - on compte près de 300 membres à La Nouvelle-Orléans comme à New York dans les loges françaises avant $1815^{40}$-, le développement de ces structures de sociabilité participe de l'essor de la conscience politique au sein de la population française. En effet, la franc-maçonnerie joue un rôle moteur dans les recompositions sociales et politiques en cours. D'une part, elle contribue à intégrer des populations d'origines diverses autour d'une fraternité dont la vocation est de dépasser les frontières nationales, sociales et politiques. D'autre part, elle constitue aux États-Unis un vecteur de développement de la culture patriotique, en référence aux pères fondateurs américains, illustres francs-maçons ${ }^{41}$. Dès leur arrivée aux États-Unis, les loges issues de Saint-Domingue ont amendé leurs règlements en signe d'adhésion aux nouvelles autorités :

À tous les banquets, les santés seront réglées ainsi qu'il suit: (voyez les changements)

La $1^{\text {e }}$ santé: "À la santé de l'Empire français de l'Empereur \& de tous les Gouvernements protecteurs des M." est remplacée par "Au Gouvernement des États-Unis, de son président et de tous les gouvernements protecteurs des Maçons" 42

19 Steven Bullock rappelle que, dans la société américaine de la jeune république, la francmaçonnerie joue un rôle politique dans les liens informels qu'elle assure entre les élites américaines ${ }^{43}$. Cette remarque peut s'étendre aux loges françaises aux États-Unis dans cette période. Sans avoir une finalité exclusivement politique - puisque les statuts maçonniques excluent souvent explicitement ces préoccupations "profanes» - elles développent néanmoins des formes d'idéal qui l'associent étroitement avec la république et les pères fondateurs, et les formes politiques « aristocratiques » en vigueur, à travers des pratiques de patronage. Ce brassage des élites est également ouvert à une culture maçonnique transatlantique, où les figures de Washington comme de Lafayette prédominent ${ }^{44}$. Cette adaptation au contexte américain n'empêche pas de garder la marque de l'héritage colonial, notamment au regard des discriminations raciales au sein des loges: les règlements de la Grande Loge de Louisiane précisent que ne peuvent prétendre à l'initiation que les individus "d'une réputation intacte, du sexe masculin, d'un âge mûr, c'est-à-dire d'avoir au moins vingt-et-un an, né de parens libres et blancs, sains de corps et de jugement, et qu'ils aient en outre des biens ou un état ou profession qui puisse leur assurer une existence honnête ${ }^{45}$ ».

Cependant, l'expression de ces solidarités est indissociable des clivages sociaux et politiques issus de l'ère des révolutions et liés à l'intégration des migrants dans les sociétés d'accueil. Les loges sont une interface tout à fait révélatrice d'une géographie maçonnique encore incertaine, à l'heure où les rites sont en constante évolution et où les autorités maçonniques traditionnelles - en France métropolitaine ou dans les colonies sont encore bouleversées par les épisodes révolutionnaires. Bien que les motifs des rivalités et des tensions entre et au sein des loges ne se lisent pas toujours très clairement, on peut tenter d'en éclairer certains aspects. D'une part, les divisions à New York semblent provenir d'une difficile agrégation des membres de l'ancienne loge 
huguenote avec les nouveaux maçons domingois, ce qui expliquerait la scission de 1805 entre «les descendants des Huguenots \& les Français ${ }^{46}$ " menant à la création de La Sincérité. Plus qu'une division religieuse - L'Union Française comme La Sincérité comptant des catholiques et des protestants en leur sein -, il semble que les clivages soient davantage sociaux et politiques. La Sincérité regroupe des militaires et aristocrates français, alors que L'Union Française est davantage une loge de négociants français et d'artisans issus de Saint-Domingue.

Les motifs de désunion à La Nouvelle-Orléans semblent être davantage liés à la question de l'autorité maçonnique et des rites au sein de l'Atlantique, bien que les divisions politiques et sociales sous-jacentes soient également présentes. La Parfaite Union et La Charité appartiennent aux réseaux maçonniques établis en relation avec la Grande Loge de Caroline du Sud implantée à Charleston. La Charité aurait même été reconstituée par des frères de $\mathrm{L} a$ Candeur établie précédemment à Charleston ${ }^{47}$. Elles pratiquent toutes deux le rite d'York, dominant aux États-Unis, alors que L'Étoile Polaire, créée par des réfugiés de Saint-Domingue, a transplanté le rite français, régularisé par le Grand Orient de France en 1803, de même que La Réunion des Coeurs. Or, la distance avec la France à l'heure des guerres napoléoniennes menace ces loges d'isolement, dans un contexte de rivalités locales. Du fait des liens établis par les francs-maçons de Saint-Domingue avec les loges de Philadelphie, où de nombreux réfugiés se sont installés dans les années 1790, la Grande Loge de Pennsylvanie vient alors suppléer aux autorités françaises. Louis Casimir Moreau Lislet, avocat et ancien propriétaire de Saint-Domingue, qui a séjourné à Philadelphie en 1794 avant de repartir sur l'île, pour la quitter définitivement en 1803, est l'acteur principal de cette adaptation des loges françaises au contexte américain. Il est à l'origine du passage des deux loges de rite français moderne, La Réunion Désirée et L'Étoile Polaire, au rite d'York entre 1808 et 1811, et de leur affiliation à la Grande Loge de Pennsylvanie. Il rédige ainsi les articles de transition de L'Étoile Polaire entre rite français et rite d'York ${ }^{48}$. Cette synthèse entre traditions française, coloniale et américaine dans le domaine maçonnique peut être mise en parallèle avec son travail de rédaction du Code civil de la Louisiane. À la demande de la Législature de la Louisiane, il a en effet été chargé en 1808, avec le juriste américain James Brown, de synthétiser les traditions juridiques espagnoles et françaises héritées de la période coloniale et du code Napoléon au sein du nouveau code civil louisianais ${ }^{49}$. Il représente également la Grande Loge de Pennsylvanie lors de la création en 1810 de deux nouvelles loges au rite d'York, La Concorde et La Persévérance. Cette logique d'intégration permet l'unification des rites et des affiliations: le rite français et l'affiliation au Grand Orient de France disparaissent temporairement pendant une décennie en Louisiane.

Les cinq loges francophones de la Nouvelle-Orléans - les deux loges anglophones ayant refusé de s'y associer - parviennent alors à s'accorder pour créer la Grande Loge de Louisiane. Cet événement est révélateur puisqu'il a lieu en 1812, l'année où la Louisiane devient État de l'Union après la phase de transition territoriale, et marque en ce sens une autonomisation locale, aussi bien politique que dans les structures maçonniques. Les premiers officiers de la Grande Loge, Pierre François Dubourg, négociant originaire du Cap-Français, et Louis Casimir Moreau Lislet, témoignent de la prééminence des réfugiés de Saint-Domingue en son $\operatorname{sein}^{50}$. Cette ascension des réfugiés traduit ainsi les évolutions de la société louisianaise, ainsi que la capacité d'intégration maçonnique en divers cercles d'appartenance qui favorisent l'implication politique dans la vie de la cité. 

atlantique, permet d'appréhender la façon dont les réfugiés de Saint-Domingue traversent les troubles révolutionnaires et s'organisent dans leurs lieux de passage ou d'installation, à New York et La Nouvelle-Orléans. Le fait que les loges s'adaptent à leur environnement, en termes de rites et d'affiliations, témoigne de la souplesse de cette forme de sociabilité qui, malgré les tensions et dissensions internes souvent à l'œuvre, prospère aux États-Unis dans les premières décennies $d u$ XIX ${ }^{e}$ siècle. Leur caractère élitiste et cosmopolite, qui s'ouvre néanmoins à un certain nombre de catégories intermédiaires, va de pair avec l'extériorisation de l'action maçonnique, à travers ses interactions avec d'autres formes de sociabilité, notamment dans le cadre religieux ou philanthropique, qui assurent un lien social aux anciens colons déracinés après l'indépendance haïtienne.

\section{NOTES}

1. Darrell R. MEADows, The Planters of Saint-Domingue, 1750-1804: Migration and Exile in the French Revolutionary Atlantic, thèse de doctorat, Carnegie Mellon University, 2004, p. 133.

2. « Morin », Encyclopédie de la franc-maçonnerie, dir. Éric SAUNIER, Paris, LGF, 2000, p. 587-589.

3. Éric SAUNIER, «L'espace caribéen, enjeu de pouvoir pour la franc-maçonnerie française ", REHMLAC, vol. 1, n 1, mai-novembre 2009, p. 42-56.

4. Voir notamment les travaux de Chloé DUflo, La franc-maçonnerie dans les villes de Guadeloupe entre 1770 et 1848. Espaces de sociabilité et parcours individuel, mémoire de Master 2, sous la direction de Bernard Gainot, IHRF, 2009.

5. Pierre-Yves BEAUREPAIRE, L'Europe des francs-maçons : XVIII ${ }^{e}-X X I^{e}$ siècles, Paris, Belin, 2002, p. 90-99

6. Éric SAUNIER, Révolution et sociabilité en Normandie au tournant des XVIII et XIX ${ }^{e}$ siècles : 6000 francsmaçons de 1740 à 1830, Rouen, Publications des Universités de Rouen et du Havre, 1999 ; Jacques DE CAUnA, L'Eldorado des Aquitains, Gascons, Basques et Béarnais aux Îles d'Amérique, Biarritz, Atlantica, 1998. Au XVIII ${ }^{\mathrm{e}}$ siècle, la majorité des loges-mères de loges domingoises sont bordelaises ou marseillaises: Élisabeth ESCALLE et Mariel GOUYON GUILLAUME, Francs-maçons des loges françaises « aux Amériques », 1770-1850 : Contribution à l'étude de la société créole, s.n., 1993. 
7. Jacques HoUdAILLE, «Quelques données sur la population de Saint-Domingue au XVIII ${ }^{\mathrm{e}}$ siècle », Population, vol. 28, n 4/5, 1973, p. 859-872.

8. Jacques DE CAUNA, L'Eldorado des Aquitains, Gascons, Basques et Béarnais aux Îles d'Amérique, op. cit. ; Mickaël Augeron et Dominique GUillemet, Champlain ou les Portes du Nouveau Monde: cinq siècles d'échanges entre le Centre-Ouest français et l'Amérique du Nord, La Crèche, Gestes Éditions, 2004 ; Henri DE CHARNISAY, L'émigration basco-béarnaise en Amérique, Biarritz, J\&D Éditions, 1996.

9. Élisabeth ESCALLE et Mariel GOUYON GUILLAUME, Francs-maçons des loges françaises aux "Amériques ", 1770-1850: Contribution à l'étude de la société créole, op. cit.; Jean-Marc VAN HILLE, Dictionnaire des marins francs-maçons, Gens de mer et professions connexes aux XVIII ${ }^{e}{ }^{X I X}{ }^{e}$ et XX ${ }^{e}$ siècles : Travaux de la loge maritime de recherche La Pérouse (collection Kronos $n^{\circ} 56$ ), Paris, L'Harmattan, 2011 ; Georges ODo, La franc-maçonnerie dans les colonies, 1738-1960, Paris, Éditions Maçonniques de France, 2001.

10. Pierre-Yves BEAUREPAIRE, « Les loges maçonniques littorales face à la politique centralisatrice des obédiences parisiennes au XVIII ${ }^{\mathrm{e}}$ siècle », dans Gérard Le Bouëdec et François Chappé (dir.), Pouvoirs et littoraux $d u X^{e}$ au Xx siècle. Actes du colloque international de Lorient (24, 25, 26 septembre 1998), Rennes, PUR, 2000, p.181-189; Pierre-Yves BEAUREPAIRE, «Fraternité universelle et pratiques discriminatoires dans la Franc-Maçonnerie des Lumières ", Revue d'Histoire moderne et contemporaine, vol. 44, n² 2, 1997, p. 195-212 ; Éric SAUNIER, « Les pratiques culturelles dans un port de traite. Les négriers et la franc-maçonnerie au Havre au XVIII ${ }^{\mathrm{e}}$ siècle », dans Marcel DORIGNY (dir.), Esclavage, résistances et abolitions Paris, Éditions du CTHS, 1999, p. 150.

11. Georges ODO, La franc-maçonnerie dans les colonies, 1738-1960, op. cit. ; Élisabeth ESCALLE et Mariel GOUYON GUILlaume, Francs-maçons des loges françaises "aux Amériques», 1770-1850: Contribution à l'étude de la société créole, op. cit.

12. Jacques HoUdAILlE, "Quelques données sur la population de Saint-Domingue au XVIII ${ }^{\mathrm{e}}$ siècle », Population, vol. 28, n 4/5, 1973, p. 859-872; Louis-Élie MOREAU DE SAINT-MÉRY, La description topographique, physique, civile, politique et historique de la partie française de Saint-Domingue, Paris, Société française d'histoire d'outre-mer, 2004.

13. Jacques de Cauna, «Autour de la thèse du complot : franc-maçonnerie, révolution et contrerévolution à Saint-Domingue, 1789-1791", dans Cécile Revauger (dir.), Lumières, n 7 , Francmaçonnerie et politique au siècle des Lumières : Europe-Amériques, 2006, p. 289-310.

14. La sociabilité au sein de cette loge a notamment été précisément étudiée dans James $\mathrm{E}$. MCCLELLAN III, «L'historiographie d'une académie coloniale: le Cercle des Philadelphes (1784-1793) ", Annales historiques de la Révolution française, $\mathrm{n}^{\circ} 320,2000$, p. 77-88.

15. Pierre-Yves BEAUREPAIRE, «Fraternité universelle et pratiques discriminatoires dans la FrancMaçonnerie des Lumières », art. cit., p. 195-212.

16. Voir notamment Luis P. MARTíN, Les francs-maçons dans la cité : les cultures politiques de la francmaçonnerie en Europe, XIX ${ }^{e}-X^{e}$ siècle, Rennes, PUR, 2000 ; Cécile REVAUGER (dir.), Lumières, $\mathrm{n}^{\circ} 7$, Franc-maçonnerie et politique au siècle des Lumières: Europe-Amériques, op.cit.; Pierre-Yves BEAUREPAIRE, «Les loges maçonniques littorales face à la politique centralisatrice des obédiences parisiennes au XVIII ${ }^{\mathrm{e}}$ siècle ", art. cit., p. 181-189.

17. Laurent DUBoIs, Avengers of the New World. The Story of the Haitian Revolution, Cambridge, Mass., Harvard University Press, 2004 ; Darrell MEADows, The Planters of Saint-Domingue, 1750-1804: Migration and Exile in the French Revolutionary Atlantic, op. cit.

18. Gabriel DEBIEN et Philip WHRIGHT, «Les colons de Saint-Domingue passés à la Jamaïque (1792-1835) », Bulletin de la Société d'Histoire de la Guadeloupe, n² 26, 1975, p. 3-217 ; Alain YAcou, L'émigration à Cuba des colons français de Saint-Domingue au cours de la Révolution, thèse de doctorat, université Bordeaux III (Bordeaux), 1975; Agnès RENAULT, D’une île rebelle à une île fidèle: les 
Français de Santiago de Cuba: 1791-1825, Mont-Saint-Aignan, Publications des universités de Rouen et du Havre, 2012, p. 67.

19. Laurent DuboIs, Avengers of the New World. The Story of the Haitian Revolution, op. cit.; David Barry GASPAR et David Patrick GEGGUS, A Turbulent Time: The French Revolution and the Greater Caribbean, Bloomington, Indiana University Press, 1997; Darrell MEADows, The Planters of SaintDomingue, 1750-1804: Migration and Exile in the French Revolutionary Atlantic, op. cit. ; Ashli WHITE, "A Flood of Impure Lava": Saint Dominguan Refugees in the United States, 1791-1820, thèse de doctorat, Columbia University, 2003.

20. Agnès RENAULT, La communauté française de Santiago de Cuba entre 1791 et 1825, thèse de doctorat, Université du Havre (Le Havre), 2007.

21. Gabriel DEBIEN, «Réfugiés de Saint-Domingue expulsés de la Havane en 1809 », Anuario de Estudios AmÉricanos, $\mathrm{n}^{\circ}$ 35, 1979, p. 555-610.

22. Ashli WhITE, " "A Flood of Impure Lava”: Saint Dominguan Refugees in the United States, 1791-1820 », op. cit.; Paul F. LACHANCE, « The 1809 Immigration of Saint-Domingue Refugees to New Orleans: Reception, Integration and Impact », Louisiana History, vol. XXIX, n 2, 1988, p. 109-141.

23. Darrell R. MEADOWS, «Engineering Exile: Social Networks and the French Atlantic Community, 1789-1809 ", French Historical Studies, vol. 23, 2000, p. 67-102; Frances SERGEANT CHILDS, French Refugee Life in the United States, 1790-1800; an American Chapter of the French Revolution, Baltimore, Johns Hopkins University Press, 1940, p. 103-121; Agnès RENAULT, « Los francmasones franceses de la jurisdiccion de Cuba al principio del siglo xix », Revista de Estudios Históricos de la Masonería Latinoamericana y Caribeña, vol. 1, $\mathrm{n}^{\circ}$ 1, 2009, p. 61-71, p. 61.

24. Allan ротоғкку, "The "Non-Aligned Status" of French Emigrés and Refugees in Philadelphia, 1793-1798 », Transatlantica, 2006, transatlantica.revues.org/1147, consulté le 30 novembre 2017.

25. Nathalie DESSENS, «Élites et diasporas : les réfugiés de Saint-Domingue dans les Amériques au $\mathrm{XIX}^{\text {ème }}$ siècle ", dans Christian Lerat (dir.), Élites et intelligentsias dans le monde caraibe, Paris, L'Harmattan, 2008, p. 43-58.

26. Élisabeth E SCALLE et Mariel GOUYON GUILlaume, Francs-maçons des loges françaises "aux Amériques" 1770-1850: Contribution à l'étude de la société créole, op. cit.

27. Fonds maçonnique, $\mathrm{FM}^{2}-561, \mathrm{BnF}$; «L'histoire de la Loge Écossaise de la Nouvelle-Orléans », Latomia, Documents Sharp vol. IV, $\mathrm{n}^{\circ} 129,2009$. Sur la franc-maçonnerie française avant 1800 à New York, voir Pierre F. DE RAVEL D'ESCLAPON, «Les loges françaises à New York au XVIII siècle », Chroniques d'Histoire Maçonnique, vol. 66, 2010, p. 29-51. Lors de la fondation de L'Étoile Polaire à La Nouvelle-Orléans en 1795, l'un des membres fondateurs, Jean-Baptiste Dezillets, ancien officier d'infanterie est « muni de certificats des $\mathrm{R} \therefore \mathrm{R} \therefore \mathrm{L} \therefore \mathrm{L} \therefore$ La Parfaite Union à l'O $\therefore$ de New York et de L'Union Parfaite à l'O $\therefore$ de la Rochelle en datte des 24 gbre $5760 \therefore$ et 10 avril $5763 \therefore$ ", Fonds maçonnique, $\mathrm{FM}^{2}-560$, BNF.

28. Le « chapitre » désigne une « loge regroupant les maçons issus des grades supérieurs à ceux d'apprenti, de compagnon et de maître ». Pierre-Yves BEAUREPAIRE, L'Europe des francs-maçons: XVIII ${ }^{e}$-XXI ${ }^{e}$ siècles, op. cit., p. 286.

29. Chapitre La Triple Union, New York, Fonds maçonnique, $\mathrm{FM}^{2}-561$, BNF.

30. Tableau de La Réunion des Cours, 1806, MSS 895, Box 6, Howard Tilton Memorial Library, Tulane University; Agnès ReNAUlt, «Los francmasones franceses de la jurisdiccion de Cuba al principio del siglo XIX ", art. cit. ; "Articles of incorporation, Statutes \& Constitutions ", La Réunion Désirée, 1806-1808, MSS 895, Box 11, Howard Tilton Memorial Library, Tulane University.

31. La Grande Loge Provinciale de Saint-Domingue séante provisoirement à New York est mentionnée sur les planches d'installation de L'Union Française, 3 janvier 1798, Fonds Maçonnique, $\mathrm{FM}^{2}-562$, BNF. Les loges d'origine des pétitionnaires à Saint-Domingue sont La Vérité, La Loge de Sion, La Bienfaisance, Les Cœurs sans Faute, La Réunion des Cœurs Franco-Américains (Orient du Cap Français), Les Amis Réunis (Orient du Port de Paix), La Parfaite Union (Orient des Cayes) ; et, 
en France, Les Cours réunis, sensibles et véridiques (Orient de Nantes), Les Vrais Amis (Orient de Sigean), L'Union Parfaite (Orient de Nancy) : liste reproduite dans Règlements Particuliers, Historique, Etc. de l'Union Française ${ }^{\circ}$ 17, New York, A. Lallemand, 1915.

32. Pierre F. DE RAVEL D'ESCLAPON, «Les loges françaises à New York au XVIII ${ }^{\mathrm{e}}$ siècle », art. cit., p. 38-48.

33. L'histoire des premiers temps de la maçonnerie a été résumée par un franc-maçon anonyme en 1828 : Manuel Maçonnique, à l'Usage des Franc-Maçons Acceptés du Rite Ancien d'York, Résidants à la Louisiane, Edwin Lyman, 1828. Il a été repris dans James B. зсот, Outline of the Rise and Progress of Freemasonry in Louisiana. From Its Introduction to the Re-Organization of the Grand Lodge in 1850, New Orleans, 1923 ; Glen Lee GREENE, Masonry in Louisiana: a Sesquicentennial History, 1812-1962, New York, Exposition Press, 1962.

34. Les loges d'origine des fondateurs sont La Sincérité à l'O $\therefore$ de Marseille, L'Union Parfaite à l'o $\therefore$ de la Rochelle, La Parfaite à l'o $\therefore$ de Nantes, La Fidélité à l'o $\therefore$ du Havre, La Vérité à l'o $\therefore$ du Cap Français, Saint-Domingue, et La Sincérité à l'o $\therefore$ de Saint Pierre, Martinique: L'Étoile Polaire, Fonds Maçonnique, $\mathrm{FM}^{2}-560$, BNF.

35. Lettre de la $\mathrm{R} \therefore \mathrm{L} \therefore$ La Parfaite Union $\mathrm{n}^{\circ} 29$ au G $\therefore$ Or $\therefore$ de France, Or $\therefore$ de La NouvelleOrléans le 14 me Jour du 1er $\mathrm{M} \therefore \mathrm{M} \therefore$ de l'an de la vraie lumière 5802 , La Parfaite Union, Fonds Maçonnique, $\mathrm{FM}^{2}-561$, BNF. La demande de constitution au Grand Orient de France ne semble pas avoir abouti.

36. Éric SAUNIER, Révolution et sociabilité en Normandie au tournant des XVIII ${ }^{e}$ et XIX ${ }^{e}$ siècles: 6000 francs-maçons de 1740 à 1830 , op. cit., p. 91.

37. Parmi de nombreux exemples tirés de la correspondance de La Persévérance à La NouvelleOrléans entre 1810 et 1812, on peut citer la lettre du frère Visinier du 3 mai 1810, demandant une exonération de quotités pour cause de difficultés financières; celle de Saintaraille du 28 juillet 1812, demandant une aide pour un frère emprisonné pour dettes; ou celle de la veuve Casalis le 26 juillet 1812, demandant l'aide pour la veuve d'un frère: MSS 895, Box 17, Howard Tilton Memorial Library, Tulane University.

38. Lettre de Pierre Pajaud à Julien Bayard, La Nouvelle-Orléans, 5 juin 1810, MSS 895, Box 17, Howard Tilton Memorial Library, Tulane University.

39. Correspondance de la loge La Persévérance avec La Parfaite Union, 28 juin 1810, MSS 895, Box 17, Howard Tilton Memorial Library, Tulane University.

40. On dénombre 315 membres initiés ou affiliés à L'Union Française à New York de 1797 à 1814, publiés dans la «Liste des membres de "L'Union Française" depuis sa fondation ", Règlements de L'Union Française $n^{\circ}$ 17, New York, s. n., 1952, p. 93-106. Il faut leur ajouter les membres de la loge La Sincérité de 1805 à 1814, dont on ne dispose pas des tableaux. Un certain nombre de francsmaçons n'appartenant pas à L'Union Française, et dont on peut supposer qu'ils appartiennent à $L a$ Sincérité, apparaissent dans les tableaux du chapitre La Triple Union dont on dispose de 1806 à 1812 : ils représentent 14 des 66 hauts grades membres du chapitre : Fonds Maçonnique, $\mathrm{FM}^{2}-561$, BNF. À La Nouvelle-Orléans, on compte 338 membres dans les loges françaises avant 1815 : Marieke POLFLIET, Émigration et politisation: les Français de New York et La Nouvelle-Orléans dans la première moitié du XIX siècle (1803-1860), Thèse de doctorat, Université de Nice-Sophia Antipolis, 2013, p. 777-816.

41. Steven C. BULLOCK, Revolutionary Brotherhood: Freemasonry and the Transformation of the American Social Order, 1730-1840, Chapel Hill, University of North Carolina Press, 1996, p. 137-162.

42. Statuts et règlements particuliers de la $\mathrm{R} \therefore \mathrm{L} \therefore \quad$ La Réunion Désirée revue, corrigés et augmentés dans ses séances des $12^{\mathrm{e}}$ du $7^{\mathrm{e}} \mathrm{M} \therefore, 21^{\mathrm{e}} \mathrm{du} 10^{\mathrm{e}} \mathrm{M} \therefore$ et $18^{\mathrm{e}}$ du $11^{\mathrm{e}} \mathrm{M} \therefore 5806$, Chapitre 14, Article 4 ; MSS 895, Box 11, Howard Tilton Memorial Library, Tulane University. 
43. «In Almost Every Place Where Power is of Importance: Politics, 1790-1826 », chapitre 8, dans Steven C. BULLOCK, Revolutionary Brotherhood: Freemasonry and the Transformation of the American Social Order, 1730-1840, op. cit., p. 220-238.

44. Les francs-maçons français de New York se sont ainsi associés aux honneurs rendus à la mort de Washington en organisant une cérémonie dans l'Église du Saint-Esprit le 31 décembre 1799 : John Albert MAYNARD, The Huguenot Church of New York: a History of the French Church of Saint Esprit, New York, 1938, p. 207

45. Ahiman Rezon ou Reglemens Généraux à l'usage des Anciens Maçons d'York, sous la Jurisdiction de la G $\therefore$ L $\therefore$ de la Louisiane, La Nouvelle-Orléans, P. et C. Roche, 1813, p. 13.

46. Victor DE LIEUVIN, « Histoire de la Loge l'Union Française $n^{\circ} 17$ ", L'Union Française - Collection de documents divers de 1795 à 1997, 1886, p. 16-17.

47. Manuel Maçonnique, à l'Usage des Franc-Maçons Acceptés du Rite Ancien d'York, Résidants à la Louisiane, op. cit., p. 2.

48. Manuel Maçonnique, à l'Usage des Franc-Maçons Acceptés du Rite Ancien d'York, Résidants à la Louisiane, op. cit., p. 5.

49. Alain A. LEVASSEUR, Louis Casimir Elisabeth Moreau-Lislet, Foster Father of Louisiana Civil Law, Baton Rouge, La., Louisiana State University, Law Center Publications Institute, 1996, p. 95-133; Alain A. LEVASSEUR, Moreau-Lislet: The Man Behind the Digest of 1808, Baton Rouge, La., Claitor's Publishing Division, 2008.

50. James B. зсот, Outline of the Rise and Progress of Freemasonry in Louisiana, op. cit., p. 19-20.

\section{RÉSUMÉS}

Cet article se propose d'analyser les conséquences des révolutions de Saint-Domingue sur les formes de sociabilité atlantiques, et plus spécifiquement la franc-maçonnerie, qui tenait une place éminente et innovante avant la Révolution. En effet, au tournant du XIx ${ }^{\mathrm{e}}$ siècle, suite à la révolte des esclaves de Saint-Domingue puis la perte définitive de l'île et l'indépendance d'Haïti, la fuite et l'implantation d'un nombre important de colons dans les grandes villes américaines suscite la recréation de loges maçonniques et leur adaptation au contexte américain. New York et La Nouvelle-Orléans accueillent, en particulier, un nombre significatif de réfugiés de SaintDomingue qui sont à l'origine de la fondation de multiples loges qui deviennent des théâtres d'expérimentation qui prolongent celles menées à Saint-Domingue, notamment en matière de rites et de formation de réseaux maçonniques. La transition entre refuge temporaire et exil définitif pour les Domingois se traduit aussi par une américanisation progressive - mais incomplète - des structures maçonniques animées par les réfugiés dans les premières décennies du XIX ${ }^{\mathrm{e}}$ siècle.

This article is about the revolutionary episodes that took place in Saint Domingue and their consequences on Atlantic forms of sociability, and more precisely on freemasonry, which held a significant and unprecedented social role before the Revolution. At the beginning of the 19th century, the slave revolt and the Haitian independence led a large number of colonists to seek refuge in major United States cities, where they recreated masonic lodges. Most notably, New York and New Orleans hosted numerous refugees, who created various lodges where they could experiment new rites and expand masonic networks. The fact that a temporary refuge turned 
into a permanent exile led to the Americanization of the masonic structures run by Domingans in the first decades of the 19th, even though such Americanization remained incomplete.

INDEX

Mots-clés : sociabilité, réfugiés, franc-maçons, Saint-Domingue, États-Unis, 19e siècle

Keywords : sociability, Refugees, Freemasons, Saint-Domingue, United States, 19th century

\section{AUTEUR}

\section{MARIEKE POLFLIET}

Université de Nice Sophia Antipolis

EHESS 